\title{
Editor's Message to Special Issue of Internet and Operation Technologies in the Era of IoT
}

\author{
KAZUYUKI YoshidA ${ }^{1, a)}$
}

IoT (internet of things) that physical objects such as sensor devices are connected to each other via a network has started to spread widely. There are limitations in size and power consumption for many of the IoT devices. For this reason, IoT devices do not have common network functions which servers or LAN switches have. So there is a need for new management techniques and security measures for them. In this special issue, we have made a concerted effort to attract excellent papers discussing problems concerned in the Internet and operation technologies in the era of IoT with a lot of experiences and practices.

This special issue is planned by the Special Interest Group on Internet and Operation Technology (SIG-IOT) as a central role. We, 14 members of editorial board, received 15 papers and reviewed them thoroughly and carefully. In the same way of former special issues arranged by SIG-IOT, this special issue also has relationship with Internet and Operation Technology Symposium (IOTS), and we include about half of the IOTS 2015 program committee members to reinforce the relationship. In this special issue, we received 3 papers based on presentation in the IOTS 2015. After cautious and careful review, we selected 4 papers (1 paper is from IOTS 2015) to be included in this special issue. In addition to these papers, this issue includes an invited paper about FESTIVAL EU-Japan collaborative project to provide a platform for developing and testing emergent Smart ICT services.

The editor in-chief wishes to express thanks to whom it may concern in IPSJ and SIG-IOT, the authors who submit excellent papers, the reviewers for careful reviews and instructive comments, and the editorial board members of this special issue for the continued encouragement, guidance and support in the editorial process of this special issue. Hopefully this special issue will be beneficial information for readers and contribute to develop academic and social infrastructure.

\author{
The Editorial Committee
}

- Editor in-Chief:

Kazuyuki Yoshida (Oita University)

- Deputy Chief Editor:

Hayato Ishibashi (Osaka City University)

- Editorial Committee:

Yusuke Doi (Preferred Networks, Inc.)

Takashi Imaizumi (Chiba University)

Hiroki Kashiwazaki (Osaka University)

Yoshiaki Kitaguchi (Kanazawa University)

Hideo Masuda (Kyoto Institute of Technology)

Kensuke Miyashita (Kyoto Women's University)

Motonori Nakamura (National Institute of Informatics)

Akinori Saitoh (Tottori University of Environmental Studies)

Shu Sakashita (Acutus Software, Inc.)

Akira Sato (University of Tsukuba)

Tesuya Shigeyasu (Prefectural University of Hiroshima)

Nariyoshi Yamai (Tokyo University of Agriculture and Technology)
$1 \quad$ Oita University, Dannoharu, Oita 870-1192, Japan
a) yoshida@oita-u.ac.jp 